ARTICLE

DOI: $10.1038 / \mathrm{s} 41467-017-02522-z$

\title{
Fungal networks shape dynamics of bacterial dispersal and community assembly in cheese rind microbiomes
}

\author{
Yuanchen Zhang ${ }^{1}$, Erik K. Kastman ${ }^{1}$, Jeffrey S. Guasto ${ }^{2}$ \& Benjamin E. Wolfe ${ }^{1}$
}

\begin{abstract}
Most studies of bacterial motility have examined small-scale (micrometer-centimeter) cell dispersal in monocultures. However, bacteria live in multispecies communities, where interactions with other microbes may inhibit or facilitate dispersal. Here, we demonstrate that motile bacteria in cheese rind microbiomes use physical networks created by filamentous fungi for dispersal, and that these interactions can shape microbial community structure. Serratia proteamaculans and other motile cheese rind bacteria disperse on fungal networks by swimming in the liquid layers formed on fungal hyphae. RNA-sequencing, transposon mutagenesis, and comparative genomics identify potential genetic mechanisms, including flagella-mediated motility, that control bacterial dispersal on hyphae. By manipulating fungal networks in experimental communities, we demonstrate that fungal-mediated bacterial dispersal can shift cheese rind microbiome composition by promoting the growth of motile over non-motile community members. Our single-cell to whole-community systems approach highlights the interactive dynamics of bacterial motility in multispecies microbiomes.
\end{abstract}


$\mathrm{M}$ ultispecies microbial communities (microbiomes) play key roles in agricultural productivity, human health, and ecosystem services ${ }^{1-3}$, but our understanding of the ecological processes and mechanisms that structure the diversity of microbiomes is still in its infancy ${ }^{4-6}$. Small-scale (micrometer-centimeter) dispersal of bacterial cells is one key ecological process that may impact the dynamics of microbial community assembly. After a propagule (cell, spore, etc.) of a microbial species colonizes a potential habitat, the ability to grow and rapidly spread may determine both the distribution and functions of that particular species within the community.

Many bacteria use active motility, via extracellular appendages or secreted metabolites, to disperse over small spatial scales up or down gradients of resources or attractants ${ }^{7,8}$. A significant body of work from just a few model bacterial species has determined the genetic and biophysical mechanisms of active bacterial dispersal, including swimming, swarming, gliding, twitching, and sliding ${ }^{8,9}$. Almost all of these studies have used monocultures of bacteria in highly simplified laboratory environments to dissect modes and mechanisms of bacterial motility. How these bacterial motility mechanisms, discovered in highly idealized laboratory systems, translate to complex multispecies microbiomes where microbes interact is largely unknown.

Changes in the abiotic and biotic environment, due to interactions with neighboring microbial species, have the potential to alter modes and mechanisms of bacterial motility and subsequent dispersal dynamics. Metabolites secreted into the environment by neighboring species may act as chemoattractants that can direct cell movement ${ }^{10,11}$ or alter quorum sensing ${ }^{10}$. Modification of the physical environment by neighboring microbes could also impact cell dispersal. Solid surfaces can exert forces on swimming cells and guide them over long distances ${ }^{12,13}$. These same forces facilitate interactions between cells in biofilms, which can result in collective cell motility and dispersal ${ }^{14,15}$.

One potentially widespread interaction that may shape the dynamics of bacterial cell dispersal is the migration of bacterial cells on fungal hyphae ${ }^{16,17}$. Multicellular filamentous fungi form mycelial networks that enable bacteria to migrate across simplified environments ${ }^{16,18}$. The specific biological and physical mechanisms underlying these interactions are not fully understood, but fungi likely maintain microenvironments that allow motile bacteria to swim and/or swarm in otherwise dry conditions ${ }^{19}$. Most previous studies characterizing fungal-mediated bacterial dispersal relied on artificial combinations of bacteria and fungi with unknown natural histories and limited ecological contexts $^{16,20,21}$. The taxonomic breadth of bacteria that can disperse on fungal networks is also poorly characterized because prior work has largely focused on a limited number of bacteria and fungi in soil systems $s^{16,19,21-23}$. Moreover, the potential contribution of these strong, pairwise bacterial-fungal interactions to the assembly of microbiomes has not been tested. Fungal networks may shape the composition of bacterial communities by promoting the dispersal and growth of motile bacteria over nonmotile community members.

Cheese rind biofilms are an ideal system for exploring the mechanisms and consequences of fungal-mediated bacterial dispersal in multispecies microbiomes. Rind biofilms form on the surfaces of cheeses that are aged in caves around the world, and several different genera of filamentous fungi commonly co-occur with motile Proteobacteria in cheese rinds ${ }^{24,25,26,27}$. Ecological dynamics in cheese rinds are easy to dissect due to the limited diversity of these microbiomes and the ability to culture most bacterial and fungal species that grow in these communities ${ }^{27}$. Previous work has demonstrated that strong bacterial-fungal interactions occur in cheese rinds ${ }^{27-29}$, but mechanisms underlying these interactions are largely unknown.
Here we report the patterns, mechanisms, and consequences of bacterial dispersal on fungal networks in cheese rind microbiomes. We focus on one common cheese rind bacterium, Serratia proteamaculans, to characterize the mechanisms of bacterial dispersal on different fungal networks. We then place these pairwise interactions in an ecological context and quantify how fungal networks can shape the composition of multispecies cheese rind communities through dispersal facilitation. Our work highlights the ability of diverse cheese Proteobacteria to disperse on fungal networks and how fungal-mediated bacterial dispersal can promote the growth of motile bacteria over non-motile community members.

\section{Results}

S. proteamaculans disperses on cheese rind fungal networks. During a culture-based survey of cheese rinds, we observed unusual streams of bacterial cells of the bacterium S. proteamaculans (strain BW106; hereafter Serratia) on hyphae of the filamentous fungus Mucor lanceolatus (strain SN1; hereafter Mucor) (Fig. 1, Supplementary Movie 1). These growth patterns suggested that Serratia used Mucor networks to disperse, possibly through the use of active motility mechanisms. To experimentally characterize this interaction, we first quantified bacterial dispersal using a co-spotting assay on standard lab media (brain heart infusion agar or BHI agar) with three different fungal networks: Mucor, Galactomyces geotrichum (hereafter Galactomyces), and a Penicillium strain closely related to P. commune (hereafter Penicillium) (Supplementary Table 1). All three fungi were isolated from cheese rinds. We chose these three fungi because they are the dominant fungi in natural and washed rind cheeses ${ }^{27,30}$, and they represent three different types of fungal networks: Mucor is a fast-growing fungus with diffuse network growth ${ }^{31,32}$, Galactomyces is also a fast-growing fungus but forms a dense

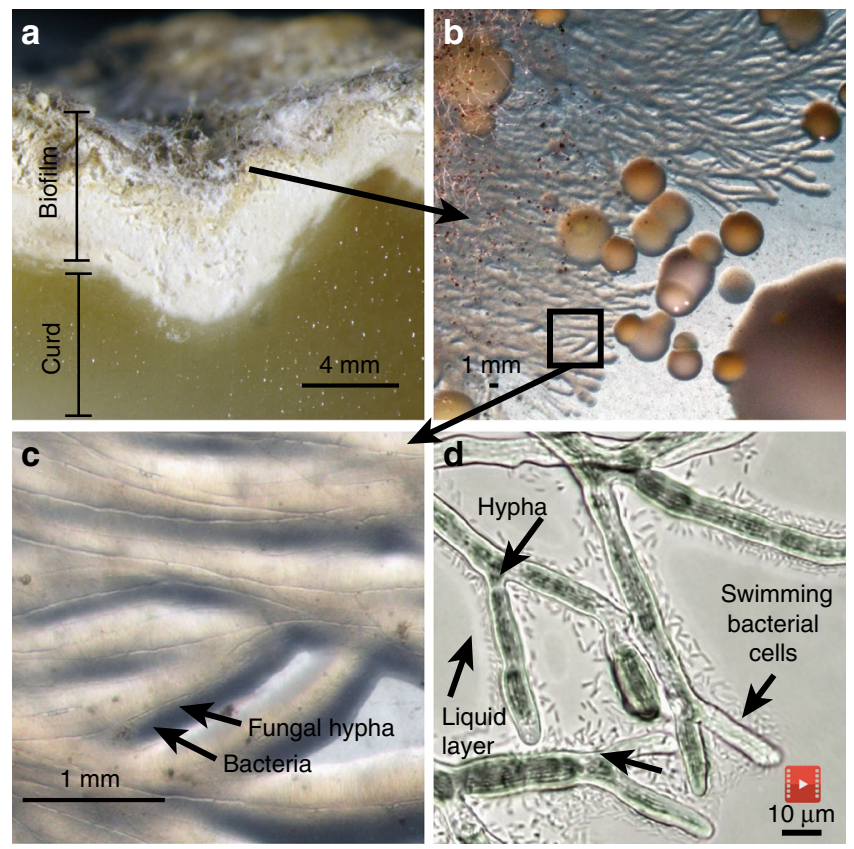

Fig. 1 Bacterial dispersal on fungal networks in a cheese rind. a Crosssection of the French cheese Saint-Nectaire, showing the curd (paste) and the biofilm (rind). b Unusual streams of bacteria were observed when plating out this rind on plate count agar with milk and salt (PCAMS). c Closer examination revealed the bacterium Serratia proteamaculans growing along the hyphae of the fungus Mucor lanceolatus. d Individual swimming Serratia cells in the liquid layer surrounding Mucor hyphae (still image from Supplementary Movie 1) 

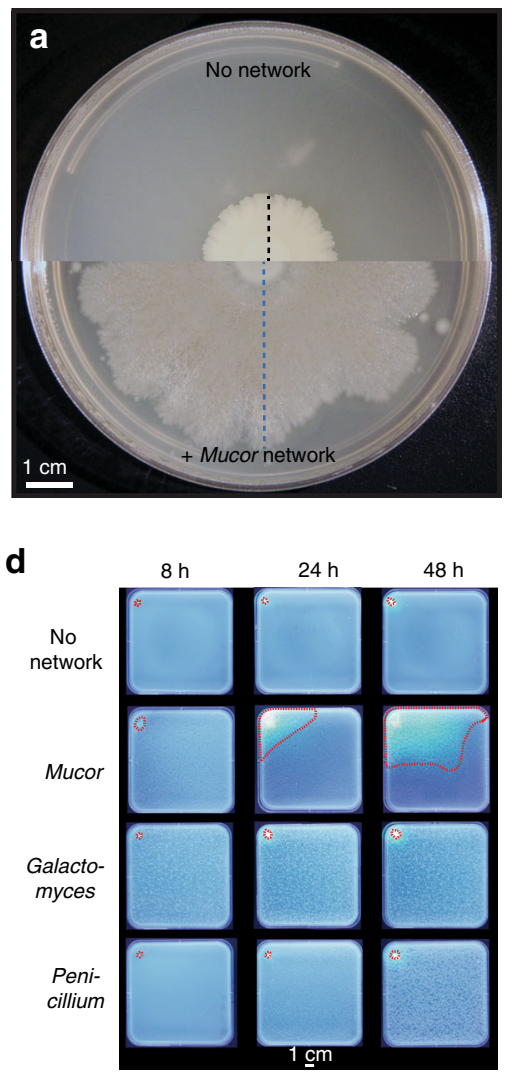

b

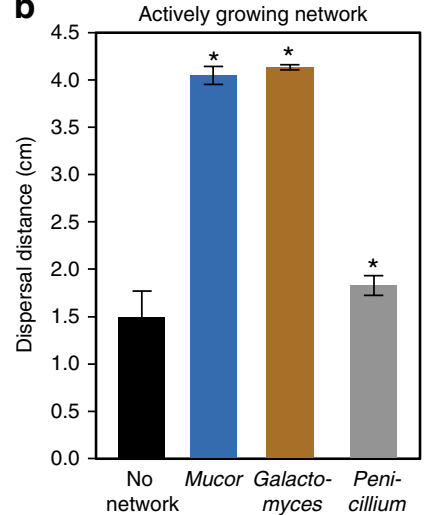

e

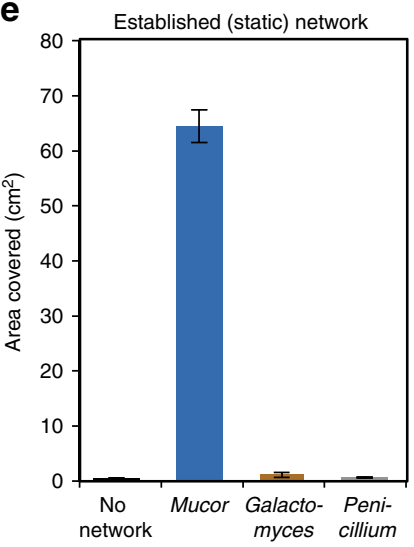

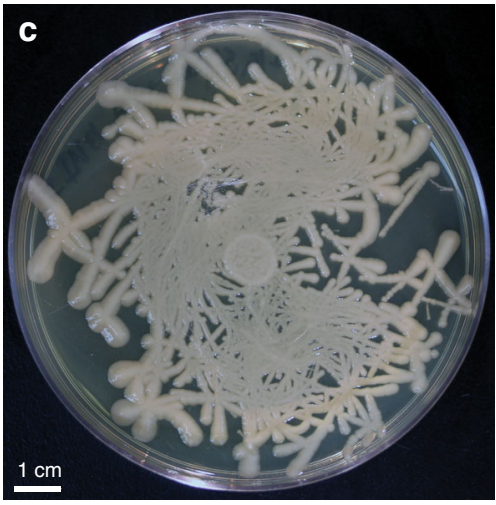

$\mathbf{f}$

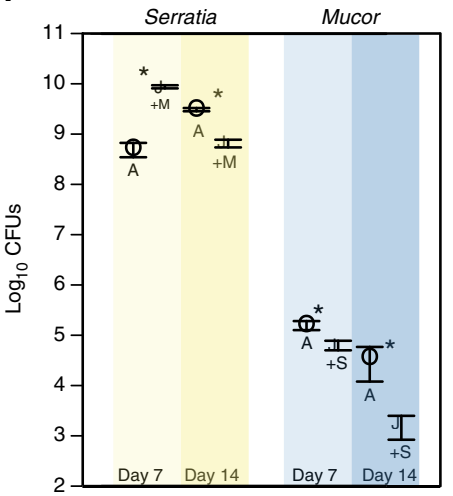

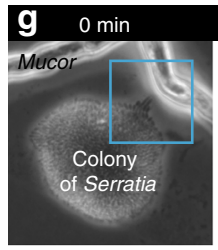
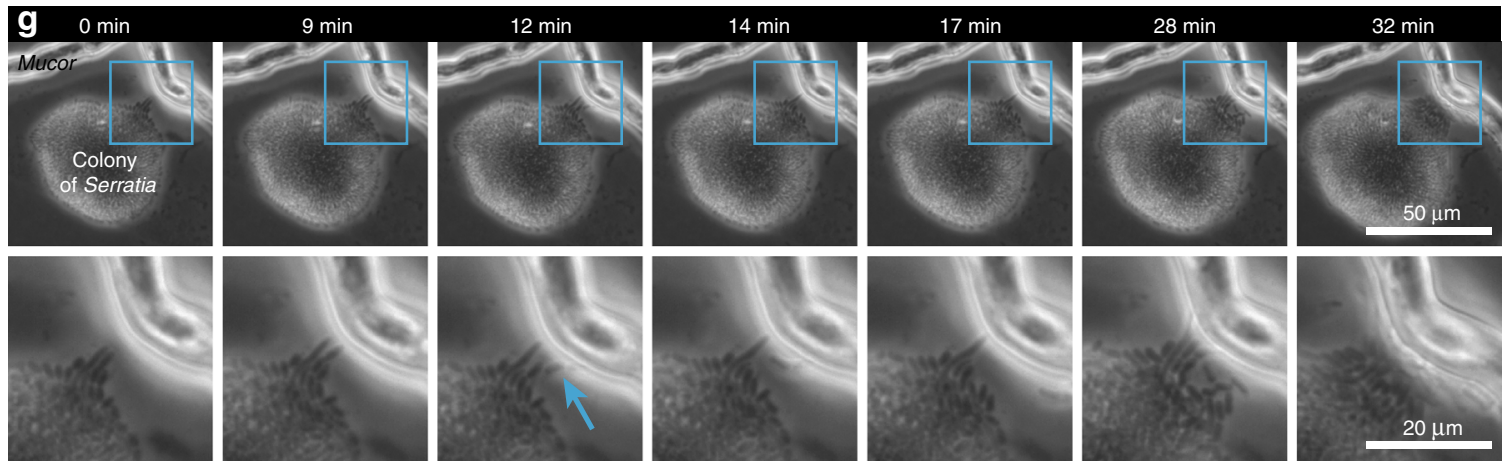

Fig. 2 Macroscopic and microscopic characterization of Serratia proteamaculans dispersal on fungal networks. a Dispersal of Serratia on PCAMS agar without Mucor (top) and with Mucor (bottom). The black dashed line indicates dispersal distance alone and the blue dashed line indicates dispersal distance with a Mucor network (as displayed in Fig. 2b). b Serratia dispersal distance after 14 days of growth. Bars show mean distance from the center of the spot to the bacterial colony edge ( \pm 1 standard deviation, $n=5)$. Asterisks indicate significant differences in dispersal compared to control (Dunnett's test, $p<0.05)$. c Dispersal of Serratia along a synthetic fungal network made of glass fibers placed on PCAMS agar. $\mathbf{d}$ Representative time-lapse images after 8,24 , and 48 hours of GFP-tagged Serratia dispersing with no network or on an already established (static) network of Mucor, Galactomyces, or Penicillium on PCAMS agar. The outermost edges of the area where Serratia spread on each fungal network are outlined in red dashed lines. Each square Petri dish is $12 \mathrm{~cm} \times 12 \mathrm{~cm}$. e Serratia dispersal distance with no network or on an established (static) network of Mucor, Galactomyces, or Penicillium. Bars show the mean area covered ( \pm 1 s.d., $n=3$ ). Area traveled on Mucor was significantly higher than the other fungal networks based on an ANOVA with a Tukey's post hoc test $\left(F_{3,15}=1788, p<0.0001\right)$. f Serratia and Mucor growth, as measured by total colony forming units (CFUs), alone and in combination, after 7 and 14 days $(n=5)$. Asterisks indicate significant differences between Alone $(A)$ and + Mucor/Serratia $(+M /+S)(S t u d e n t ' s t$-test, $p<0.05)$. $\mathbf{g}$ Time lapse of Serratia-Mucor interaction over 32 minutes. The full colony of Serratia is shown in the top row, with two Mucor hyphae at the top. The bottom row is a zoomed-in section (outlined in blue) showing a close-up of pioneer Serratia cells colonizing the Mucor hyphae, indicated by the blue arrow. See Supplementary Figure 4 for more examples

network $^{32,33}$, and Penicillium is slow-growing and forms very dense fungal networks ${ }^{32,34}$. The cells of Serratia were co-spotted on BHI agar (1.5\% agar) with each of these fungi or without a fungus ("No network", Fig. 2a). Fungal networks grew out from the co-spot, and Serratia was able to spread on the networks. After 14 days of incubation, the horizontal dispersal distance of the bacterial colony from co-spot center to colony edge was quantified using a bacterial transfer approach (see Methods).

Serratia rapidly spread on networks of both Mucor and Galactomyces, with a $173 \%$ and $179 \%$ increase, respectively, in dispersal distance across the agar surface compared to Serratia without a fungal network (Fig. 2b). In contrast, Penicillium 
networks provided limited dispersal facilitation of Serratia, with only a $23 \%$ increase in dispersal. The strong dispersal facilitation of Serratia was not limited to the environment on BHI agar; Serratia spread on Mucor networks on a range of media types, including cheese curd agar (CCA) (Supplementary Fig. 1). To confirm that this dispersal trait was not unique to our cheese strain, we quantified the ability of closely related Serratia strains and species from other environments (Supplementary Table 1) to spread on networks of Mucor. Most Serratia isolates showed substantial dispersal facilitation on Mucor networks, ranging from 145 to $175 \%$ increases in dispersal distance (Supplementary Fig. 2). Limited dispersal in a few isolates (e.g., S. proteamaculans strain B-41156, with only a $41 \%$ increase in dispersal distance) indicates natural variation in the ability of Serratia species to disperse on fungal networks.

Hyphae of filamentous fungi, including Mucor species, grow from the tip ${ }^{35}$, and bacterial dispersal on fungal hyphae could be a result of passive dispersal when bacteria are pushed horizontally across surfaces by the growing fungi. To determine if Serratia spreads using active motility or passive dispersal by the fungus, a synthetic glass fiber network was created on top of a $10-\mu \mathrm{L}$ spot of Serratia cells on BHI agar. These glass fibers are comparable in diameter $(8 \mu \mathrm{m})$ to the hyphae of Mucor $(10-25 \mu \mathrm{m})$ and provided a similar physical network for the movement of motile Serratia cells. After a week of growth, Serratia spread out from the initial spot and followed the topology of the synthetic network (Fig. 2c), suggesting that active motility drives the movement of this bacterium across physical networks.

In nature, bacterial cells may initially land in a microenvironment where an existing fungal mycelium is available for colonization. To determine if Serratia could disperse across established (static) fungal networks, we transformed S. proteamaculans BW106 with a green fluorescent protein (GFP)producing plasmid to allow for real-time, non-destructive tracking of its spread across Mucor, Galactomyces, and Penicillium networks. Unlike the assays above that just track linear dispersal distance, this approach allowed us to track the network area covered by Serratia. As with the co-spot assays above, Serratia rapidly spread across existing networks of Mucor, covering an average of $64 \mathrm{~cm}^{2}$ within $48 \mathrm{~h}$, compared to 1 and $0.6 \mathrm{~cm}^{2}$ on Galactomyces and Penicillium networks, respectively (Fig. 2d, e). The limited dispersal on static networks of Galactomyces contrasts with the high dispersal facilitation observed on actively growing Galactomyces networks (Fig. 2b). This discrepancy suggests that passive dispersal resulting from bacterial cells being pushed or dragged during fungal growth may contribute to dispersal on Galactomyces networks while dispersal on Mucor networks is largely due to active motility processes.

The substantial increase in dispersal distance of Serratia on Mucor networks may provide a significant benefit by allowing it to colonize unoccupied niches. But, changes in dispersal distance across a surface may not completely reflect the total impact on Serratia growth, as increased dispersal may actually decrease cell density. Moreover, bacterial dispersal data do not capture impacts on the fungal host. To measure the growth of both interacting partners, we determined the total colony-forming units (CFUs) of both Serratia and Mucor at 7 and 14 days of growth alone and in co-culture. As predicted from dispersal experiments, Mucor networks have a strong positive effect on Serratia growth at day 7, but this effect diminishes and results in growth inhibition at day 14 (Fig. 2f). The dispersal of Serratia on Mucor networks negatively impacts fungal growth at both day 7 and day 14 . Surprisingly, unlike most other examples where Serratia species almost entirely eradicate the fungi with which they interact ${ }^{36-38}$, Mucor is only partially inhibited by the bacterium and not completely killed (Supplementary Fig. 3).
Studies of bacterial dispersal on fungi have largely focused on macroscopic patterns (millimeter-centimeter scale) of bacterial and fungal hyphae growth ${ }^{16,21,36}$. But the initial phases of colonization occur at the micron scale and the relevant biophysical interactions that regulate bacterial dispersal on hyphae at these scales are poorly characterized. To identify how Serratia cells interact with Mucor networks at a microscopic scale, we used time-lapse microscopy of the two microbes co-cultured on a thin layer of BHI agar ( $\approx 0.7 \mathrm{~mm}$ in height). This approach revealed that interactions are initiated between individual cells of Serratia and a liquid layer surrounding Mucor hyphae (Fig. 2g). After imaging numerous bacterial-fungal contacts, we were able to consistently observe three phases of interaction initiation. First, the bacterial colony comes into physical contact with the liquid layer that surrounds the fungal hyphae as the hyphae grow near bacterial colonies. Next, pioneer cells at the edge of the bacterial colony rapidly transition from a stationary state to a motile state and swim in the liquid layer around the fungal hyphae. These pioneer cells swim along the fungal hyphae until they encounter physical barriers or reach an intersection and move to other hyphae. In the final phase, a mass of swimming cells colonizes the fungal hyphae. These interaction phases were commonly observed across replicate Serratia-Mucor contacts (Supplementary Fig. 4; Supplementary Movies 2 and 3).

The rapid cellular switch from a static colony state to a motile swimming state demonstrates that Serratia cells can quickly change behavior in the presence of the liquid layer around fungal networks. Collectively, these experiments and observations characterizing Serratia-fungal interactions from cheese rinds demonstrate that Serratia species can rapidly disperse on fungal networks via active bacterial motility mechanisms. We next sought to identify the mechanisms controlling these interactions and their ecological consequences for the assembly of these communities.

Mechanisms driving Serratia dispersal on Mucor networks. To determine potential genetic mechanisms that drive fungalmediated dispersal facilitation of bacterial cells, we used three complementary approaches: (1) transcriptome sequencing (RNAseq) to identify genes that are differentially expressed across the Serratia genome when grown on fungal networks, (2) transposon mutagenesis to identify genes that are essential for the dispersal phenotype described above, and (3) comparative genomics of different Serratia strains with variable dispersal abilities on Mucor networks. Given the rapid change from a stationary to motile cell population observed above, we predicted that the genes that control quorum sensing, flagellar biosynthesis, and other motility-related processes would be differentially expressed when Serratia was co-cultured with Mucor and would be essential for these interactions.

The presence of the fungal networks caused a shift in global gene expression of Serratia (Fig. 3a; Supplementary Data 1), with 108 genes showing significantly decreased expression and 41 genes with increased expression levels when Serratia was grown with Mucor networks. Surprisingly, the most differentially expressed genes were related to metabolic processes and other functions, not motility or quorum sensing (Fig. 3b). Of the 67 genes with decreased expression that had predicted functional annotations, almost half (33 genes) were predicted phage proteins. Significantly lower expression of genes associated with carbohydrate metabolism (8), amino acids and derivatives (4), and membrane transport (8), as well as genes associated with folate and biotin metabolism (4), suggests that growth on Mucor networks alters the supply of nutrients and vitamins available for Serratia. Surprisingly, we also detected downregulation of many 
a

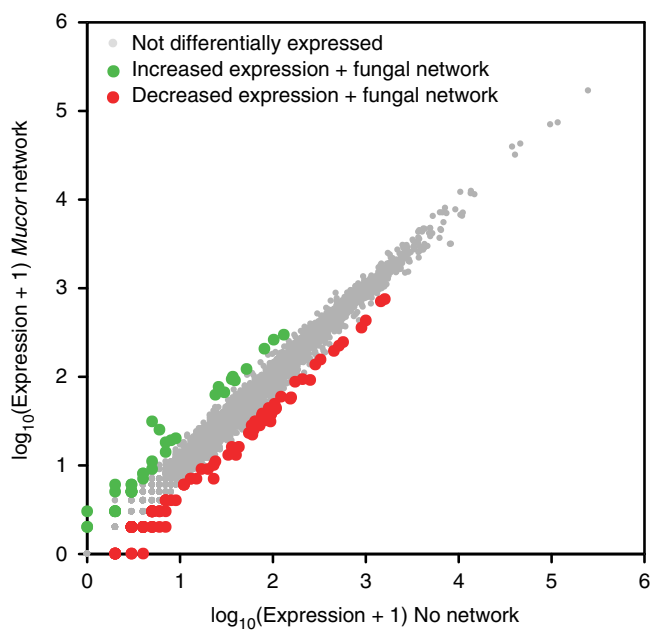

b

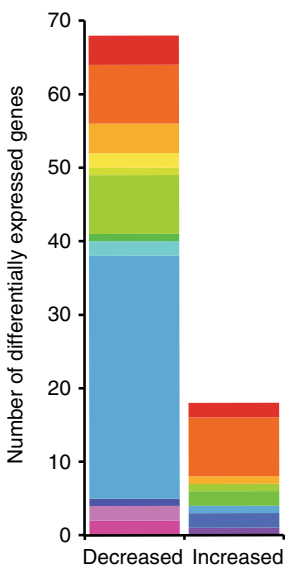

Amino acids and derivatives

- Carbohydrates

Cofactors, vitamins, pigments

Fatty acids, lipids, and isoprenoids

Iron acquisition and metabolism

Membrane transport

Metabolism of aromatic compounds

Nitrogen metabolism

Nucleosides and nucleotides

Phage

Protein metabolism

Regulation and cell signaling

Respiration

Stress response

Virulence, disease and defense

C

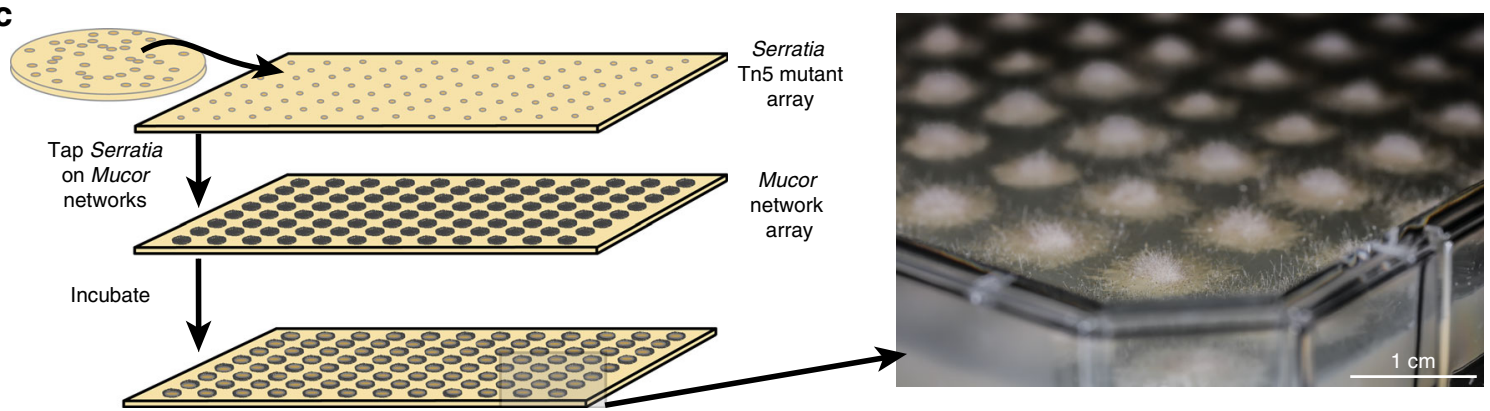

Fungal network dispersal phenotype screen

d
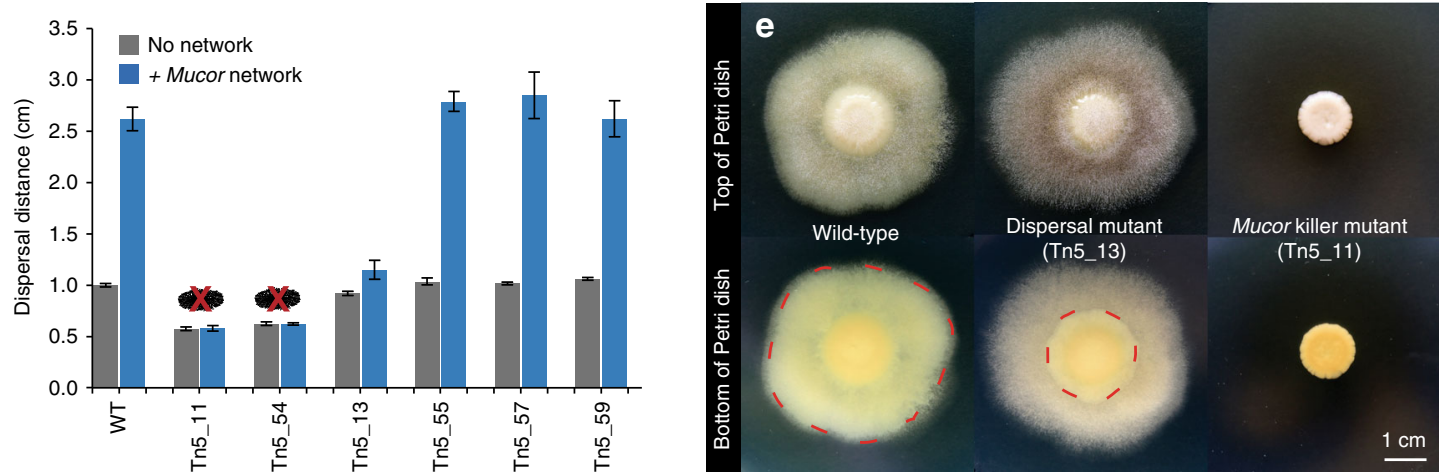

Fig. 3 Genetic mechanisms driving Serratia proteamaculans dispersal on Mucor networks. a Biplot of mean gene expression data from an RNA-seq experiment of Serratia with and without Mucor networks. Genes that had greater than 2-fold change in expression and deemed significantly different based on a $q$-value of $<0.05$ ( $p$-values adjusted for false discovery rate using the Benjamini-Hochberg procedure) were considered significantly increased or decreased in expression. Genes increased in expression are indicated in green, decreased in red, and no change in gray. Three biological replicates were performed for each treatment. b Functional distribution of differentially expressed genes that were annotated with a putative function. Functions were determined based on SEED annotations from RAST. Decreased = significantly decreased expression on Mucor networks. Increased = significantly increased expression on Mucor networks. c Illustration of the Tn 5 transposon mutagenesis screen for Serratia dispersal on Mucor. Serratia mutants were arrayed and spotted onto an existing Mucor array growing on PCAMS agar and the resultant plate was screened for changes in the dispersal phenotype. A photograph of an example screen plate is on the right (photo by Scott Chimileski and used with permission). d Dispersal distances along Mucor hyphae of putative Serratia Tn5 mutants compared to the wild-type Serratia, after 7 days of growth. Mutants Tn5_11 and Tn5_54 killed the fungal host and are marked with red "X" over mycelia. One mutant, Tn5_13, displayed limited dispersal along the fungal hyphae. Bars are mean distance from the center of the spot to the bacterial colony edge ( \pm 1 standard deviation, $n=5$ ). Dispersal assays were conducted on BHI agar. e Images of the wild-type Serratia, the dispersal deficient Tn5_13, and a killer mutant Tn5_11 with and without Mucor growing on PCAMS agar. The wild-type Serratia disperses to the edge of the hyphae, as outlined in red, while the dispersal deficient Tn5_13 does not disperse as far, as outlined in red. Mutant Tn5_11 completely inhibits the growth of Mucor. See Supplementary Fig. 6 for more genetic and phenotypic data from the selected transposon mutants 
of the predicted chitinase genes in the Serratia genome when growing on chitin-rich fungal networks (Supplementary Data 1). Homologs of these genes have been associated with antifungal properties in other Serratia species ${ }^{39,40}$ and downregulation of chitinases may partly explain why Serratia does not completely kill Mucor. Many of the genes with increased expression levels were associated with carbohydrate catabolism (Fig. 3b), again suggesting that growth on fungal networks alters the metabolism of Serratia.

A single time point of RNA-seq data cannot capture dynamic transcriptional responses that may occur during the different stages of microbial interactions. However, the overall pattern of limited differential expression of $S$. proteamaculans BW106 when grown with Mucor aligns with a previous RNA-seq study of the bacterium Serratia plymuthica grown in the presence of the fungus Rhizoctonia solani ${ }^{41}$. In that study, only 38 genes were differentially expressed, similar to the magnitude of differentially expressed genes observed in our study.

Transposon mutagenesis provides a complementary approach to RNA-seq by identifying specific genes necessary for dispersal on fungal hyphae. We used a Tn5 transposon mutagenesis system $^{42}$ to generate Serratia mutants that were then screened on arrays of Mucor networks (Fig. 3c). Using this approach, we initially identified 59 mutants that demonstrated altered colony appearance or dispersal phenotypes on Mucor networks, ranging from complete lack of dispersal on fungal hyphae to killing of the fungal host (Fig. 3d). These 59 mutants were further re-screened for fungal-mediated dispersal using the co-spotting assay described above, and six mutants with distinct phenotypes were selected for whole-genome sequencing to identify transposon insertion sites.

In our dispersal assay, the most striking mutant was Tn5_13, which was entirely dispersal deficient on both Mucor networks (Fig. 3d) and low-agar medium (Supplementary Fig. 5), suggesting a loss of motility. Using whole-genome sequencing, we discovered that the Tn5 transposon had disrupted the fliS gene in mutant Tn5_13 (Supplementary Fig. 6). FliS has not been wellcharacterized in Serratia species, but in other bacteria, FliS is a flagellin-specific chaperone that coordinates export of flagellin from the cell ${ }^{43}$. Disruption of this key regulator of flagellin biosynthesis leads to the production of short flagella and loss of motility in Bacillus subtilis and Salmonella typhimurium ${ }^{44,45}$ and may play similar roles in Serratia. Over 40 genes are predicted to be involved with flagellar biosynthesis and regulation in the $S$. proteamaculans BW106 genome. Screening 6886 mutants provided $\approx 1 \times$ coverage of the predicted genes in the $5.6 \mathrm{Mb}$ genome of S. proteamaculans BW106. More subtle loss-of-function flagellar mutants may have been difficult to identify using our macroscopic phenotypic approach. Despite this limitation, our screen supports previous targeted knockout studies and confirms a key role of flagella in fungal-mediated bacterial dispersal ${ }^{18,36,46}$.

Other mutants were motile and did not display the same striking loss of dispersal on fungal networks as Tn5_13 (Supplementary Fig. 5), but they did display altered interaction outcomes or dispersal phenotypes (Supplementary Fig. 6) that provided further insights into other genes that may impact the outcomes of Serratia dispersal on Mucor networks. Surprisingly, mutants Tn5_11 and Tn5_54 completely killed Mucor, and thus Serratia did not have a fungal network present to facilitate dispersal across the agar surface (Fig. 3e, Supplementary Fig. 6). The Tn5 transposon inserted into a predicted ADP-heptose synthase in Tn5_11 and into a predicted ferric-binding periplasmic protein in the enterobactin operon in mutant Tn5_54. Why transposon insertions in these two genes caused Serratia to kill Mucor is unclear, but disruption of metabolic pathways associated with these gene products may have resulted in the accumulation of metabolites with antifungal activity.

Three other mutants-Tn5_55, Tn5_57, and Tn5_59-formed Serratia-Mucor co-spots with altered colony edges or thicknesses (Supplementary Fig. 6), but the overall dispersal distance of these mutants on Mucor did not significantly change (Fig. 3d). These mutants had transposon insertions in genes related to phosphate metabolism (PhoU protein in Tn5_55) ${ }^{47}$, a gene with an unknown function (in Tn5_57), and a gene known to be essential in phospholipid biosynthesis (a glycerol-3-phosphate dehydrogenase in Tn5_59) ${ }^{48}$, suggesting that phosphate and phospholipid metabolism of Serratia can impact colony formation on Mucor networks.

To further investigate potential genetic mechanisms underlying Serratia dispersal on Mucor networks, we compared the genomes of the three closely related $S$. proteamaculans strains that showed different dispersal patterns on Mucor: BW106 and B-41162, which disperse on Mucor, and B-41156, which does not (Supplementary Fig. 2). Of the 62 gene annotations absent in the genome of B-41156, but present in BW106 and B-41162, one stood out: the gene fliQ, which is part of the fliLMNOPQR flagellar biosynthesis operon (Supplementary Data 2). In motile species of the Enterobacteriaceae, FliQ is one of the six transmembrane proteins that make up the flagellar export apparatus ${ }^{49,50}$. A frameshift deletion in fliQ of B-41156 leads to predicted loss of function (Supplementary Fig. 7). This loss of function was supported with a motility assay: while strains BW106 and B-41162 showed rapid dispersal across high motility $(0.6 \%$ agar $)$ plates $(515 \%$ and $525 \%$ increase in growth, respectively), strain B-41156 showed a limited increase in growth (152\%) (Supplementary Fig. 8). While other genomic differences could also contribute to the loss of spreading on fungal networks by B-41156, this observation reinforces the important role of flagella and motility in dispersal facilitation of bacteria by fungal networks.

Fungal networks shape cheese rind microbiome composition. Previous studies have described the potential existence of fungalmediated bacterial dispersal in soil systems through pairwise interaction studies of a few laboratory strains ${ }^{16,19,36}$. Whether these interactions, which were studied in isolation, can shape the composition of multispecies communities has not been determined. Strong pairwise interactions may be dampened by multispecies interactions that can occur in communities with three or more species ${ }^{51}$. Given our observation that motility is required for Serratia to disperse on fungal networks, we predicted that fungal-mediated bacterial dispersal would be unevenly distributed across cheese rind bacteria: other motile Proteobacteria species would disperse on fungal networks while non-motile Actinobacteria and Firmicutes species would have limited dispersal on fungal networks. We also predicted that this uneven dispersal facilitation would have consequences for the assembly of multispecies communities, with motile Proteobacteria favored over other bacterial taxa in communities when dispersal-promoting fungal networks were present.

Using the same co-spot approach described above with Serratia, we screened 22 cheese rind bacterial isolates spanning 13 bacterial genera and three phyla (Proteobacteria, Firmicutes, and Actinobacteria) for their ability to disperse on fungal networks. The genera of Proteobacteria screened, including Hafnia, Halomonas, Providencia, Pseudomonas, Psychrobacter, and Vibrio, generally have actively motile cells that swim or swarm using flagella ${ }^{52-57}$, while the Firmicutes and Actinobacteria genera (Staphylococcus, Arthrobacter, Brevibacterium, Brachybacterium, Corynebacterium, Leucobacter, and 


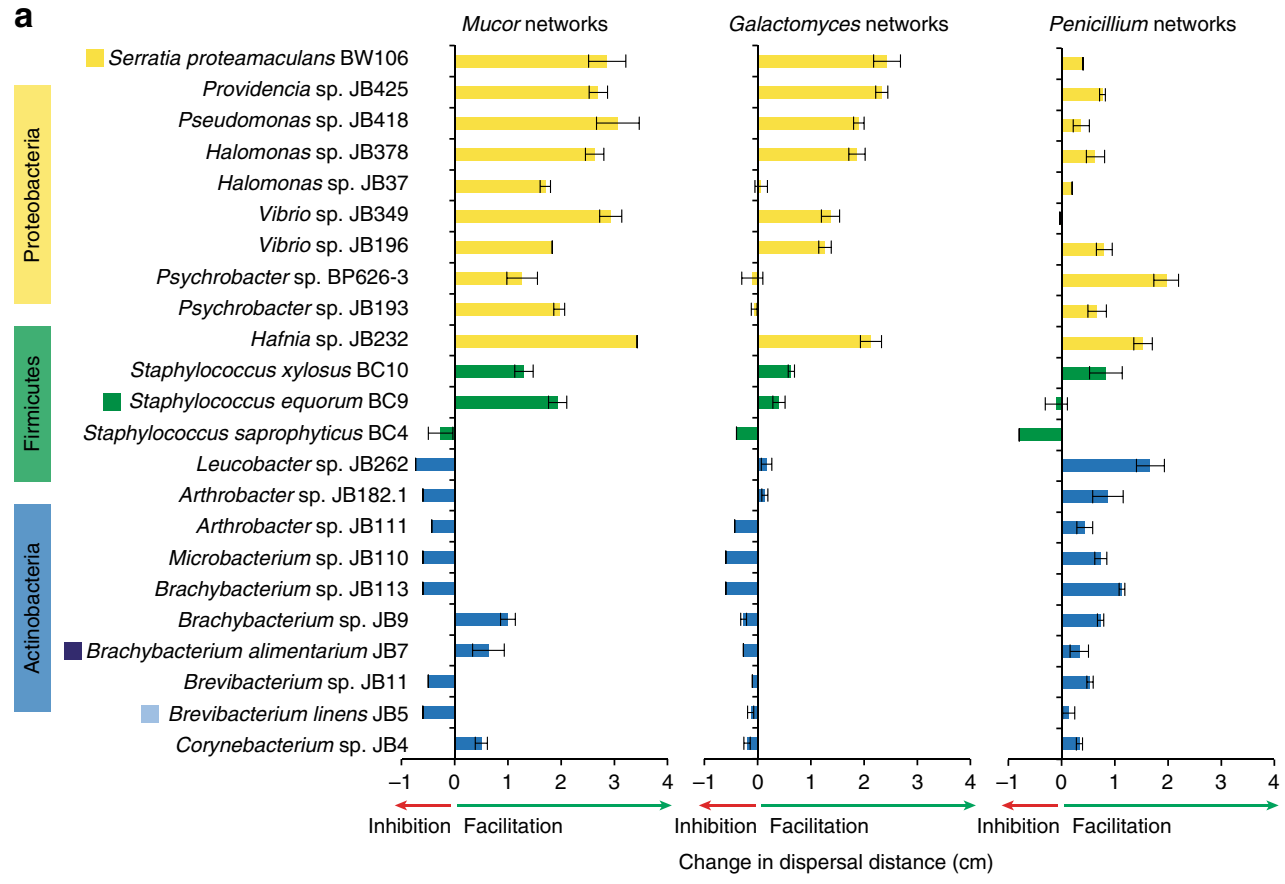

b
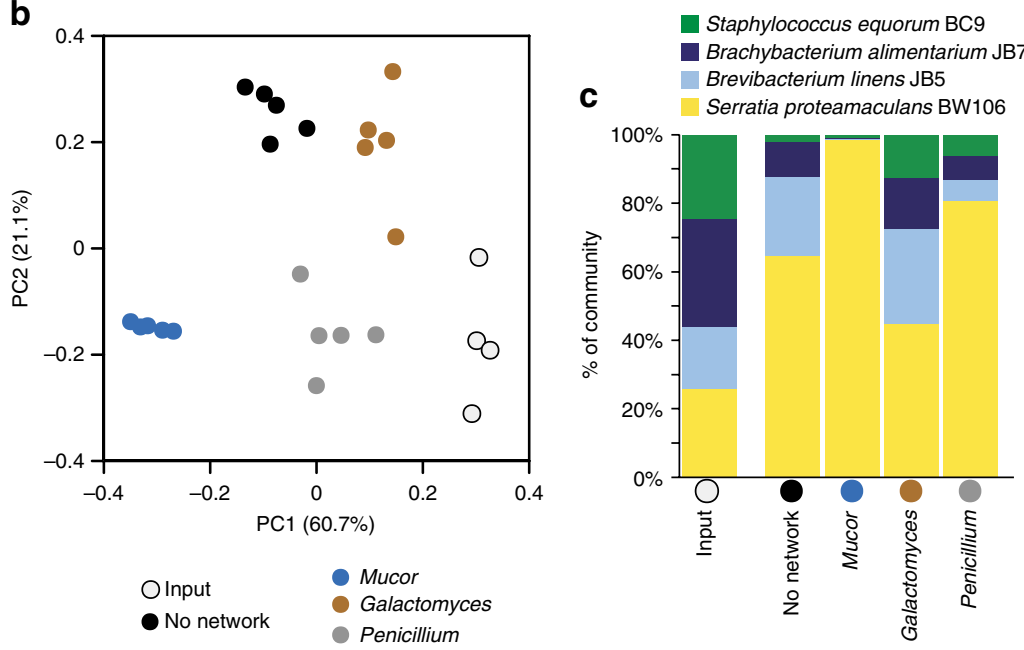

d

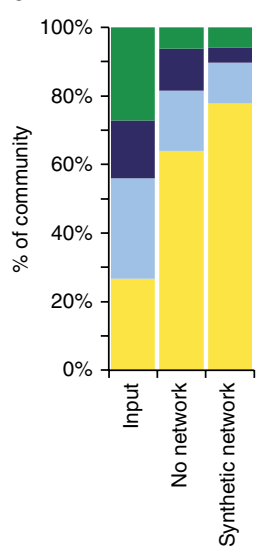

Fig. 4 Fungal networks facilitate the dispersal of Proteobacteria and shape the diversity of cheese rind communities. a Dispersal of various cheese rind bacteria on fungal networks. Data show change in dispersal distance (as measured from the center of the bacterial colony to the edge) on Mucor, Galactomyces, or Penicillium networks compared to growth alone after 14 days of growth. Dispersal assays were conducted on BHI agar. Bars are mean \pm 1 standard deviation $(n=3)$. Taxa indicated with a colored box were used in community experiments. $\mathbf{b}$ Principal coordinates analysis of relative abundance data from experimental communities on cheese curd agar with different fungal networks. "Input" indicates the input inoculum used to set up the experiment. The "No network" treatment included no fungus. Mucor, Galactomyces, and Penicillium treatments included living fungal networks of the respective fungi. Each community treatment was replicated five times. The addition of fungal networks shifted the composition of the bacterial communities compared to no network communities (PERMANOVA $F=72.14, p<0.001$ ). c Mean relative abundance (\% of total CFUs) of bacteria within the experimental communities with different fungal networks after 14 days of growth. Treatments are the same as in $\mathbf{b}$. $\mathbf{d}$ Mean relative abundance (\% of total CFUs) of bacteria in control communities ("No network") and communities with a glass fiber network ("Synthetic network") after 14 days of growth. Synthetic networks shifted bacterial community composition compared to the no network controls (PERMANOVA $F=7.38, p<0.01$ )

Microbacterium) are considered non-motile ${ }^{58-63}$. Fungal networks strongly facilitated the dispersal of Proteobacteria compared to Firmicutes and Actinobacteria on Mucor (nested ANOVA $F_{2,65}=13.80, p<0.001$; Fig. $4 \mathrm{a}$ ) and Galactomyces networks $\left(F_{2,65}=9.94, p<0.01\right.$; Fig. $\left.4 \mathrm{a}\right)$, but there were no differences across phyla on Penicillium networks $\left(F_{2,65}=2.97, p=\right.$ 0.074; Fig. 4a) networks. This pattern of differential dispersal facilitation across bacterial phyla strongly supported our prediction that most motile Proteobacteria can disperse on fungal networks, while Firmicutes and Actinobacteria cannot.
To test whether fungal networks can impact cheese rind microbiome diversity by promoting the growth of Proteobacteria, we inoculated CCA with equal CFUs of $S$. proteamaculans BW106 (Proteobacteria-high dispersal on fungal networks), Staphylococcus equorum BC9 (Firmicutes-medium dispersal on fungal networks), Brevibacterium linens JB5 (Actinobacteria-low dispersal on fungal networks), and Brachybacterium alimentarium JB7 (Actinobacteria-low dispersal on fungal networks) as well as the yeast Debaryomyces hansenii. The yeast was included because it is a common component of cheese rind microbial 


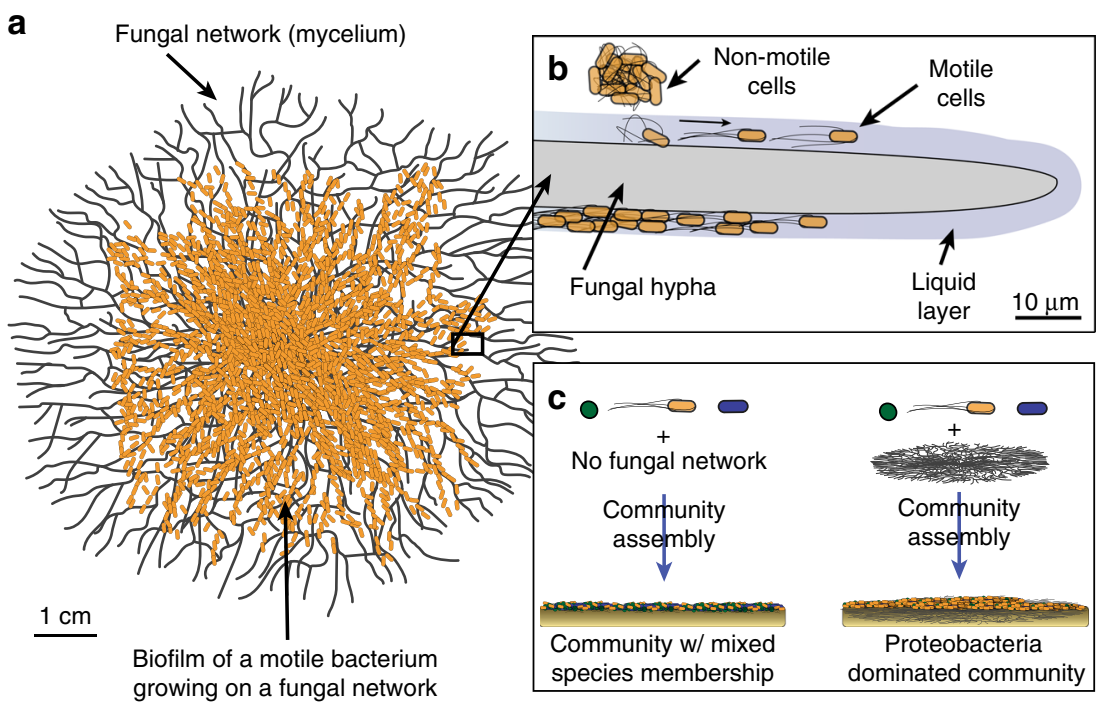

Fig. 5 Overview of mechanisms and consequences of fungal-mediated bacterial dispersal in cheese rind microbiomes. a Cells of motile Proteobacteria spread across fungal networks, leading to increased dispersal across cheese surfaces. b Serratia proteamaculans, and likely other motile Proteobacteria, use flagella to swim in the liquid layers surrounding Mucor hyphae. c The presence of fungal networks in cheese rind communities leads to the promotion of motile Proteobacteria growth over non-motile community members

communities and it facilitates the deacidification of the cheese curd medium ${ }^{27}$. Four different fungal treatments were then applied to these experimental communities: (1) No network, where no fungus was inoculated, (2) Mucor, (3) Galactomyces, and (4) Penicillium. Fungi were added as spores and were allowed to form mycelia as they grew with the bacterial communities. After 2 weeks of growth, a rind had formed on the cheese surface and the experimental communities were harvested to determine CFUs of each bacterium present.

As predicted, the addition of fungal networks shifted the composition of the bacterial communities compared to no network communities (PERMANOVA $F=72.14, \quad p<0.001$; Fig. 4b), with Mucor communities having the highest relative abundances of Serratia across all treatments (No network: $64.9 \pm$ 2.1\%; Mucor: $98.5 \pm 0.3 \%$; Galactomyces: $45.2 \pm 3.1 \%$; Penicillium: $80.1 \pm 3.0 \%$; ANOVA $F_{3,19}=89.2, p<0.001$; Fig. $\left.4 c\right)$. The limited impact of Galactomyces on community composition is surprising, given that this fungus strongly promoted the dispersal of Serratia alone (Fig. 4a), and indicates that pairwise interactions cannot always predict outcomes in multispecies communities. Surprisingly, Penicillium caused an increase in the relative abundance of Serratia in the experimental communities even though it demonstrated limited dispersal facilitation in co-culture experiments. Previous work in this system demonstrated that Penicillium inhibits the growth of Brevibacterium and Brachybacterium to a greater extent than it does Staphylococcus or Serratia, possibly due to the production of antibacterial compounds $^{27}$, and this differential inhibition may be driving the shift in bacterial composition when Penicillium is present.

To tease apart the relative impact of dispersal of bacteria on networks versus other abiotic or biotic interaction mechanisms, we repeated the same experiment but instead added glass fiber networks described above to create synthetic networks on the cheese surface (Supplementary Fig. 9). We predicted that the relative abundance of Serratia would increase in the presence of synthetic networks as we observed in the Mucor treatments. After 2 weeks of growth, the synthetic networks had shifted in composition (PERMANOVA $F=7.38, p<0.01$; Fig. $4 d$ ), with significantly higher relative abundance of Serratia compared to the no network control treatment (ANOVA $F_{1,13}=9.25, p<0.01$; No network: $64.1 \pm 9.9 \%$; Synthetic network: $77.8 \pm 6.6 \%)$. The more limited effects of the synthetic networks on community composition compared to living Mucor networks (Fig. 4c) could be because synthetic networks do not grow with the bacterial populations over time and/or because biotic cues or conditions generated by the fungi are missing. Regardless, these data demonstrate that the presence of physical networks alone can cause bacterial communities to shift in composition through differential dispersal facilitation of bacterial species.

\section{Discussion}

Fungi and bacteria co-occur in many types of microbiomes, including animal hosts, soils, and food systems ${ }^{17}$. Despite the potential for substantial diversity of bacterial-fungal interactions in such microbiomes, the biology of most bacterial species has been studied without considering the potential role of fungi as mediators of evolutionary and ecological processes. We demonstrate that strong, pairwise interactions between bacteria and fungi can not only shape the small-scale dispersal dynamics of a single bacterial species, but can also impact the diversity of multispecies bacterial communities (Fig. 5). The results presented here-in concert with recent studies from other systems $28,64-66$ suggest that a thorough understanding of mechanisms regulating microbiome assembly requires both eukaryotic and prokaryotic perspectives.

It has been previously demonstrated that motile bacteria can migrate on fungal hyphae ${ }^{16,18,20,21,36}$, but studies of the genetic mechanisms of these interactions are limited and are often based on the selection of a few candidate genes ${ }^{36}$. Our novel interaction-based transposon mutagenesis screen used an untargeted approach to identify any non-essential genes controlling this interaction. We determined that flagella-mediated motility is essential for the dispersal of $S$. proteamaculans in liquid layers on fungal hyphae (Fig. 5b). Previous studies of fungal-mediated bacterial dispersal have not tested the effect of these pairwise interactions in the context of multispecies communities. Using the tractable cheese rind model microbiome ${ }^{25,27,28}$, we demonstrated that fungal-mediated bacterial dispersal can shape the composition of relatively simple multispecies microbiomes by promoting the growth of motile Proteobacteria (Fig. 5c). This dispersal facilitation was not limited to a single strain of 
bacterium, but was also identified in numerous strains of $S$. proteamaculans and Serratia liquefaciens as well as a range of other Gammaproteobacteria species. We acknowledge that our cheese rind microbial communities are relatively low in species diversity, and the impacts of fungal-mediated bacterial dispersal interactions may be more diffuse in complex communities. But given that Mucor and other filamentous fungi often co-occur in environments with motile Proteobacteria ${ }^{67-69}$, we predict that this biophysical interaction can play key roles in determining the composition of other microbiomes.

Many aspects of the diversity, mechanisms, and impacts of these interactions remain to be explored. How do co-occurring motile bacteria interact and compete on fungal networks? Do motile pathogenic species, such as Listeria monocytogenes, use fungal networks to disperse across cheese surfaces and other environments? Given the high cost of producing flagella ${ }^{18}$, can fungal networks impact the evolution of motility traits in bacteria? Future work using the cheese rind model microbiome and other tractable systems will continue to explore the causes and consequences of fungal-mediated bacterial dispersal.

\section{Methods}

Isolation and maintenance of cultures. Strains were isolated from the rinds of cheeses by serially diluting cheese rind scrapings on plate count agar with milk and salt, or PCAMS ${ }^{27}$. For all experiments described below, strain BW106 of S. proteamaculans isolated from the Saint-Nectaire cheese described above was used with strain SN1 of M. lanceolatus (isolated from the same cheese). Inocula for all experiments were created from frozen glycerol stocks of bacterial overnight cultures grown in BHI broth for $16 \mathrm{~h}$. These experimental glycerol stocks were plated to determine CFUs per $\mu \mathrm{L}$ of inoculum, and these CFU densities were used to standardize the inputs into the experiments below. Fungal stocks were created by either scraping the surface of a plate containing spores (Mucor and Penicillium) or from liquid overnight cultures grown in yeast peptone dextrose broth (Galactomyces).

To comprehensively identify the species of Serratia and Mucor isolated from Saint-Nectaire, as well as Serratia strains received from the United States Department of Agriculture ARS Culture Collection (NRRL), we used wholegenome sequencing to create draft genomes as previously described ${ }^{28}$. Draft genome sequences have been deposited in NCBI (see Supplementary Table 1 for accession info). To construct a phylogenomic tree of the Serratia species, we first identified single-copy genes shared by all Serratia species from RAST-annotated ${ }^{70}$ genomes that were assembled using CLC Genomic Workbench. An alignment of all single-copy genes was made using $\mathrm{MUSCLE}^{71}$, and then a maximum likelihood tree was constructed using RAxML ${ }^{72}$ using the General Time Reversible + Gamma (GTRGAMMA) model. To place the Mucor strain SN1 isolated from cheese within a phylogenetic context, we used previously published 18S rRNA, 28S rRNA, ITS, and rpb1 sequences from Mucor isolated from cheese and other environments ${ }^{73}$. Using a low coverage $(10 \times)$ assembly of reads from a 100 -bp, paired-end, genomic library of Mucor strain SN1 (Supplementary Table 1), we extracted 18S rRNA, 28S rRNA, ITS, and rpb1 genes for this phylogeny. An alignment of concatenated sequences from representative strains was used to construct a maximum likelihood phylogeny using RAxML (Supplementary Fig.10).

Network dispersal co-spot assays. To measure the dispersal of Serratia on actively growing fungal networks, 250 CFUs of Serratia were inoculated in $10 \mu \mathrm{L}$ of $1 \times$ phosphate buffered saline (PBS) onto the center of a BHI agar (1.5\% agar) plate with 500 CFUs of a fungus (Mucor, Galactomyces, and Penicillium networks) or with no fungus (No network). Bacterial and fungal cells used in the co-spots were from frozen glycerol stocks with a known number of CFUs/ $\mu \mathrm{L}$. The $10-\mu \mathrm{L}$ spot of PBS dried within a few minutes of adding it to the plate and did not impact the growth or dispersal of the bacterial cells. We used an initial ratio of 1:2 Serratia to Mucor based on pilot experiments where this ratio gave most consistent outputs and because final bacterial and fungal densities using this approach reached levels similar to those found in cheese rinds ${ }^{74,75}$. Plates were incubated at $24^{\circ} \mathrm{C}$ for 14 days.

In addition to BHI agar, various other types of media were used to demonstrate that this interaction is not specific to one medium type (Supplementary Fig. 1). These media included $\mathrm{CCA}^{27}$, PCAMS ${ }^{27}$, potato dextrose agar (PDA), and yeast extract sucrose agar (YES). Most experiments in the manuscript were conducted on BHI, PCAMS, or CCA. Because the hydration level of different types of media can impact the motility of bacteria ${ }^{18,23}$, we used media that had similar water activity $\left(a_{\mathrm{w}}\right)$ to what is found in fresh cheese curds (BHI $a_{\mathrm{w}}=0.992$; PCAMS $a_{\mathrm{w}}=0.992$; CCA $a_{\mathrm{w}}=0.972$; fresh cheese curds $a_{\mathrm{w}}=0.988$ ).

To quantify the extent of bacterial dispersal across the fungal network, we developed a transect "tap and transfer" method. A sterile toothpick, measuring the radius of the Petri dish $(4.2 \mathrm{~cm})$, was tapped on the fungal network from the center of the plate (center of the initial spot of bacterial and fungal cells) to the outside edge of the actively growing fungal mycelium. The toothpick was then removed from the experimental plate and tapped onto the surface of a new BHI agar plate containing cycloheximide $(50 \mathrm{mg} / \mathrm{mL})$ to inhibit fungal growth for Penicillium and Mucor networks and natamycin $(21.6 \mathrm{mg} / \mathrm{L})$ for Galactomyces, which is resistant to cycloheximide. Transfer plates were incubated for $24 \mathrm{~h}$ at $24^{\circ} \mathrm{C}$ and the length of the transects with Serratia growth was used to infer the distance traveled across the fungal network. ANOVAs were used to determine significant differences in dispersal distance across the fungal network treatments.

Synthetic fungal networks, made of glass fibers, were used to determine if active motility was necessary for dispersal on fungal networks. After a $10-\mu \mathrm{L}$ spot of Serratia inoculum in PBS containing 250 CFUs absorbed into BHI agar, sterile glass fibers were placed on the surface of the agar at a similar density to living fungal networks. Glass fibers have a similar thickness $(8 \mu \mathrm{m})$ to that of fungal hyphae, which range from 4 (Penicillium) to $25 \mu \mathrm{m}$ (Mucor) in diameter ${ }^{73,76}$. After 2 days of growth, the presence of dispersal across the synthetic network was noted and representative plates were photographed.

To determine if $S$. proteamaculans could spread on existing fungal networks, we inserted a plasmid (pGLO, BioRad) containing a gene for GFP with an araC promoter into S. proteamaculans BW106. Electrocompetent cells of $S$. proteamaculans were made by first growing up overnight cultures for $16 \mathrm{~h}$ in liquid BHI medium. The cultures were then diluted 100-fold in liquid BHI medium in a baffle flask. The cultures were grown with agitation for $2-4 \mathrm{~h}$ until the $\mathrm{OD}_{600}$ measured 0.5. The culture was chilled on ice for $15 \mathrm{~min}$, then centrifuged at $3000 \times \mathrm{g}$ and $4{ }^{\circ} \mathrm{C}$ for $10 \mathrm{~min}$. The resulting pellet was washed four times at decreasing volumes $(125,75,25$, and $5 \mathrm{~mL})$ of $10 \%$ glycerol. After the final wash, the cells were re-suspended in $2.5 \mathrm{~mL} 10 \%$ glycerol and frozen at $-80^{\circ} \mathrm{C}$ for at least $18 \mathrm{~h}$. These cells were used for electroporation at $1.8 \mathrm{kV}, 25 \mu \mathrm{F}$, and $200 \Omega$, to transform the strain with the pGLO plasmid.

Square Petri dishes $(12 \mathrm{~cm} \times 12 \mathrm{~cm})$ containing PCAMS with ampicillin (100 $\mathrm{mg} / \mathrm{mL})$ and arabinose $(2 \mathrm{mg} / \mathrm{mL})$ were inoculated with 10,000 CFUs of either Mucor, Galactomyces, or Penicillium. Plates were incubated at $24^{\circ} \mathrm{C}$ for $48 \mathrm{~h}$ before inoculum of $S$. proteamaculans BW106 containing the pGLO plasmid was tapped into the top left corner of the plate using the tip of a sterile toothpick. Each fungal network treatment and a control treatment (No network) were replicated four times. After $48 \mathrm{~h}$ of growth when the fungal hyphae had formed a complete lawn across the plates, the area colonized by Serratia was determined by photographing plates while exposed to a long-wave UV lamp. ImageJ was used to trace outlines of the area colonized by Serratia and to quantify the total area colonized. ANOVAs were used to determine significant differences in area colonized across the fungal network treatments.

Co-culture growth assays. Mucor growth was quantified by co-inoculating Mucor (500 CFUs) and Serratia (250 CFUs) at the center of a Petri dish. Controls of Mucor (500 CFUs) in $10 \mu \mathrm{L}$ PBS and Serratia (250 CFUs) in $10 \mu \mathrm{L}$ PBS were also created. Each treatment was replicated five times each for two time points: 7 and 14 days. At each time point, the population was quantified using a whole plate harvest technique. With a sterile pipette tip, the agar and microbes were excised from the plastic Petri dish and placed into a $710-\mathrm{mL}$ Whirl-Pak ${ }^{\otimes}$ bag with $30 \mathrm{~mL}$ of $1 \times$ PBS. The agar and microbes were manually homogenized by rolling a closed 50 $\mathrm{mL}$ conical tube across the outside of the bag to pulverize and mix the agar with the PBS. The homogenate was serially diluted on selective media (BHI agar with cycloheximide $100 \mu \mathrm{g} / \mathrm{mL}$ for bacteria and BHI agar with chloramphenicol $50 \mu \mathrm{g} /$ $\mathrm{mL}$ for fungi). The CFUs on these plates were counted to quantify the abundance of Serratia and Mucor. We chose CFU quantification and not other measures of bacterial and fungal abundance (biomass) because it is a commonly used measure of bacterial and fungal abundance in cheese rinds and systems with easy to culture microbes, it is a metric that can be easily used across both bacteria and fungi, and it captures the total number of reproductive units in both types of microbes. We acknowledge that in Mucor, CFUs can originate from both hyphal fragments and spores.

Microscopy. Thin layers $(\approx 0.7 \mathrm{~mm}$ in height $)$ of $\mathrm{BHI}$ agar $(1.5 \%$ agar) were poured into $60 \mathrm{~mm} \times 15 \mathrm{~mm}$ Petri dishes on a level surface and left to set for $4 \mathrm{~h}$. Serratia and Mucor were co-inoculated in the center of the Petri dish in a 5- $\mu \mathrm{L}$ spot of liquid $1 \times$ PBS containing $115 \mathrm{CFUs} / \mu \mathrm{L}$ of Serratia and $1150 \mathrm{CFUs} / \mu \mathrm{L}$ of Mucor. This ratio of Serratia and Mucor cells provided an ideal distribution of Serratia and Mucor colonies for imaging initial contacts between the bacterium and fungus. The Petri dishes were incubated at room temperature $\left(24^{\circ} \mathrm{C}\right)$ in the light for at least 10 $\mathrm{h}$ prior to imaging. Images and movies of Serratia-Mucor interactions were taken on a Nikon TiE inverted microscope using phase contrast imaging with an Andor Zyla 5.5 camera under $40 \times$ magnification ( $0.6 \mathrm{NA}$ ). Three replicate Serratia-Mucor contacts were imaged on multiple days to determine the robustness of phases of cell contact and growth. Three replicate time-lapse examples are presented in the manuscript.

RNA-seq. Experimental populations of $S$. proteamaculans BW106 growing alone (No network) and on Mucor networks (+ Mucor network) were constructed by inoculating circular $100-\mathrm{mm}$ Petri dishes containing $20 \mathrm{~mL}$ of PCAMS ${ }^{27}$. Plates 
were inoculated with 5000 CFUs of S. proteamaculans (No network) or a mix of 5000 CFUs of S. proteamaculans and 5000 CFUs of Mucor (+ Mucor network) and incubated at $24^{\circ} \mathrm{C}$ for $27 \mathrm{~h}$. Each treatment was replicated three times. Cells were harvested by scraping the agar surface with a sterile razor blade to remove most of the microbial biomass. Harvested cells were stored in RNAProtect Reagent (Qiagen) to stabilize mRNA and frozen at $-80^{\circ} \mathrm{C}$. RNA was extracted using a standard phenol-chloroform protocol described previously ${ }^{77}$. This protocol uses a standard bead-beating step in a lysis buffer to release cell contents from pelleted cells in RNAProtect. To ensure that the RNA was of high quality and not degraded, $500 \mathrm{ng}$ of each RNA prep was run and visualized on a 1.5\% agarose gel. DNA was removed from the nucleic acid pool using a TURBO DNA-free kit (Life Technologies). 5S rRNA and tRNA were depleted using MEGAClear (Life Technologies) kits. 16S and 23S rRNA were depleted using RiboZero (Illumina) kits. To remove both fungal and bacterial ribosomal RNA, yeast and bacterial rRNA probes from the RiboZero kits were mixed 1:2 and used for rRNA depletion. To confirm that the samples were free of DNA contaminants, a PCR of the 16S rRNA was conducted with standard $16 \mathrm{~S}$ primers (27f and 1492r).

RNA-seq libraries were constructed from purified mRNA using the NEBNext Ultra RNA Library Prep Kit for Illumina (New England Biolabs) using manufacturer's instructions and sequenced using paired-end $100 \mathrm{bp}$ reads on an Illumina HiSeq Rapid Run by the Harvard Bauer Core Facility. About 16 million reads were sequenced for each library. Only forward reads of the paired ends were used for analysis. Raw reads and differential expression data have been submitted to the NCBI GEO database. The project is available as GEO Series Accession Number GSE85095. Analysis of RNA-seq libraries, including read mapping, normalization, and quantification of transcript abundances, was done using Rockhopper version $1.3 .0^{78}$ with default settings as previously described ${ }^{28}$. The $S$. proteamaculans BW106 genome was concatenated and used as the reference genome for read mapping. It has been deposited at DDBJ/ENA/GenBank under the accession MCGS00000000. Expression values were normalized by the upper quartile of gene expression. We considered differentially expressed genes to be those that were greater than 2-fold change in expression when comparing No network to + Mucor network replicates. Expression differences were deemed statistically significant based on a $q$-value of $<0.05$. Rockhopper's $q$-values are $p$ values adjusted for false discovery rate using the Benjamini-Hochberg procedure. Functional assignment of differentially expressed genes was determined from the

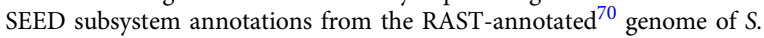
proteamaculans BW106.

Transposon mutagenesis. To identify genes that impact Serratia dispersal on fungal networks, we used the EZ-Tn $5^{\mathrm{TM}}<\mathrm{KAN}-2>$ Tnp Transposome ${ }^{\mathrm{TM}}$ Kit (Epicentre) to generate a transposon mutant library. Electrocompetent cells (as described above) were used to electroporate the $\mathrm{EZ}-\mathrm{Tn} 5^{\mathrm{TM}}$ Transposome into Serratia. Cells were plated onto PCAMS containing $50 \mu \mathrm{g} / \mathrm{mL}$ kanamycin to select for successful transformants. Colonies were then patched onto Nunc Omni Trays in an $8 \times 12$ grid to match a 96 -pin replicator.

To screen the mutant library for altered dispersal phenotypes on fungal networks, each 96-array of mutants was tapped onto an array of Mucor networks that had been growing for $24 \mathrm{~h}$. Mucor networks were generated by tapping the 96pin replicator onto a lawn of three-day-old Mucor to collect spores, which were then transferred to a fresh Omniplate containing PCAMS with $50 \mu \mathrm{g} / \mathrm{mL}$ kanamycin. This array of 96 spots of Mucor was incubated at $24^{\circ} \mathrm{C}$ for $24 \mathrm{~h}$, after which a 96-array of $S$. proteamaculans mutants was inoculated on the networks using the same 96-pin method. After another $24 \mathrm{~h}$ of growth, the resulting cocultures were visually screened using $4 \times$ magnification under a dissecting microscope. We confirmed that lack of dispersal was not the result of poor growth by also tapping the mutant library on Omni Trays without Mucor. A subset of putative mutants was isolated and rescreened using the co-spot assay described above in "Network dispersal co-spot assays".

Transposon insertion sites were determined by using whole-genome sequencing of the putative mutants. DNA was extracted using MoBio PowerSoil DNA extraction kits from streaks generated from a single bacterial colony grown for $2-3$ days on BHI agar. Approximately $1 \mu \mathrm{g}$ of purified gDNA was sheared using NEBNext dsDNA fragmentase (New England Biolabs) to a size range of approximately 300-700 bp NEBNext Ultra DNA Library Prep Kit for Illumina (New England Biolabs). Libraries were spread across multiple sequencing lanes with other projects and were sequenced using 100-bp, paired-end reads on an Illumina HiSeq 2500. Approximately 10 million reads were sequenced for each genome. Failed reads were removed from the libraries and reads were trimmed to remove low-quality bases and were assembled to create draft genomes using the de novo assembler in CLC Genomics Workbench 8.0. Insertion sites were identified by generating a BLAST searchable database of the whole genome and then searching for the transposon sequence.

Putative mutants were also screened for loss of motility by spotting 250 CFUs of cells in $10 \mu \mathrm{L}$ of $1 \times$ PBS in the center of a Petri dish. Each mutant was screened for motility using five biological replicates. Plates were incubated at $24^{\circ} \mathrm{C}$, and the radius of bacterial dispersal was measured after 14 days of growth. protein coding genes of that strain to two closely related motile strains, BW106 and B-41162. Core and accessory genes were identified using PGAP ${ }^{79}$, as previously described for Staphylococcus species ${ }^{28}$. Species-to-species orthologs were identified by pairwise strain comparison using BLAST with PGAP defaults: a minimum local coverage of $25 \%$ of the longer group and a global match of no less than $50 \%$ of the longer group, a minimum score value of 50 , and a maximum $\mathrm{E}$ value of $1 \mathrm{e}-8$. Multistrain orthologs were then found using MultiParanoid in PGAP.

Community experiment. To determine how fungal networks might impact the dispersal of other bacterial taxa that live in cheese rinds, the same network dispersal co-spot assay described above for S. proteamaculans BW106 was used to measure fungal-mediated bacterial dispersal of an additional 22 bacterial isolates spanning the phyla Proteobacteria, Firmicutes, and Actinobacteria (Supplementary Table 1). These bacteria are commonly the dominant species in rind communities found on Saint-Nectaire cheese and other natural and washed rind cheeses ${ }^{27}$. As with co-spot experiments above, bacteria were spotted alone or with each of the three fungal networks (Mucor, Galactomyces, and Penicillium). Based on low variation observed across biological replicates in pilot experiments, three biological replicates were conducted for each treatment. The dispersal distance from the center of the co-spot to the edge of the bacterial colony was measured using the same transect and tap method described above. Data are expressed as change in dispersal distance when on a network compared to alone. Nested ANOVAs were used to test for significant differences in dispersal distances on fungal networks between the phyla Proteobacteria, Firmicutes, and Actinobacteria.

An in vitro community reconstruction approach was used to determine how fungal networks impact bacterial community composition. CCA was used to grow a model cheese rind community consisting of $B$. linens JB5, B. alimentarium JB7, $S$. proteamaculans BW106, and S. equorum BC9 (see Supplementary Table 1 for strain origin information). The yeast $D$. hansenii $135 \mathrm{~B}$ was also added to these communities because it is widespread in cheese rinds and facilitates deacidification of the cheese $\mathrm{curd}^{27}$. Three fungal networks treatments were applied to this community: Mucor, Galactomyces, or Penicillium. As with co-spots above, bacterial cells and fungal spores used in this experiment came from $-80^{\circ} \mathrm{C}$ glycerol stocks with a known number of CFUs/ $\mu \mathrm{L}$. All species were mixed in approximately equal concentrations ("Input") for a total CFU of 20,000. A control community with no fungal network ("No network") was also created by adding the same volume of $1 \times$ PBS used for the fungal network inocula. The communities were plated onto the surface of $20 \mathrm{~mL}$ of CCA dispensed in $100 \mathrm{~mm}$ Petri dishes. During this experiment, fungal networks grew with the bacterial cells on the surface of the cheese curd to form a rind. Experimental units were incubated in the dark for 4 days at $24^{\circ} \mathrm{C}$ and then for 10 days at $14^{\circ} \mathrm{C}$ in order to mimic the conditions of a cheese cave. After 2 weeks, each community was homogenized in $1 \times$ PBS, serially diluted, and then plated onto PCAMS media for the quantification of each species. All four bacteria have unique colony morphologies and pigments (Supplementary Fig. 11) and can be easily distinguished from one another.

To isolate the effect of physical networks for growth and dispersal of bacterial cells from other chemical or biological effects of the fungal networks, we repeated the experiment with a synthetic glass fiber network treatment. The same input inoculum described above containing B. linens JB5, B. alimentarium JB7, S. proteamaculans BW106, S. equorum BC9, and the yeast D. hansenii 135B was added to 14 CCA plates. To seven replicate plates, $1 \mathrm{~g}$ of the synthetic glass fiber network described above was spread across the surface of the agar after the inoculum had dried onto the cheese curd surface ("Synthetic network", Supplementary Fig. 9). The remaining seven replicate plates were not manipulated and served as controls ("No network"). Experimental units were incubated in the dark for 4 days at $24^{\circ} \mathrm{C}$ and then at $14^{\circ} \mathrm{C}$ for 10 days as above. Bacterial community composition was determined by counting colonies as described above.

In both the fungal network and synthetic network experiments, the differences in community composition across treatments were determined with PERMANOVA and changes in the relative abundance of Serratia across treatments were determined using ANOVA. Principal coordinates analysis was used to visualize differences in community composition across replicates using the BrayCurtis dissimilarity index.

Data availability. Draft genome sequences for Serratia and Mucor strains have been deposited in the NCBI BioSample database (accession codes are listed in Supplementary Table 1). The S. proteamaculans BW106 draft genome has been deposited in the DDBJ/ENA/GenBank databases under accession code MCGS00000000. RNA-seq transcriptomic data have been deposited in the NCBI Gene Expression Omnibus (GEO) database under accession code GSE85095. All other relevant data are included in this published article and its Supplementary Information files, or can be obtained from the corresponding author upon request.

Received: 6 April 2017 Accepted: 7 December 2017

Published online: 23 January 2018 


\section{References}

1. Cho, I. \& Blaser, M. J. The human microbiome: at the interface of health and disease. Nat. Rev. Genet. 13, 260-270 (2012).

2. Philippot, L., Raaijmakers, J. M., Lemanceau, P. \& van der Putten, W. H. Going back to the roots: the microbial ecology of the rhizosphere. Nat. Rev. Microbiol. 11, 789-799 (2013).

3. Gibbons, S. M. \& Gilbert, J. A. Microbial diversity-exploration of natural ecosystems and microbiomes. Curr. Opin. Genet. Dev. 35, 66-72 (2015).

4. Nemergut, D. R. et al. Patterns and processes of microbial community assembly. Microbiol. Mol. Biol. Rev. 77, 342-356 (2013).

5. Shade, A. Diversity is the question, not the answer. ISME J. 11, 1-6 (2017).

6. Konopka, A., Lindemann, S. \& Fredrickson, J. Dynamics in microbial communities: unraveling mechanisms to identify principles. ISME J. 9, 1488-1495 (2015).

7. Ben-Jacob, E., Finkelshtein, A., Ariel, G. \& Ingham, C. Multispecies swarms of social microorganisms as moving ecosystems. Trends Microbiol. 24, 257-269 (2016).

8. Kearns, D. B. A field guide to bacterial swarming motility. Nat. Rev. Microbiol. 8, 634-644 (2010).

9. McBride, M. J. Bacterial gliding motility: multiple mechanisms for cell movement over surfaces. Annu. Rev. Microbiol. 55, 49-75 (2001).

10. Waters, C. M. \& Bassler, B. L. Quorum sensing: cell-to-cell communication in bacteria. Annu. Rev. Cell Dev. Biol. 21, 319-346 (2005).

11. Sourjik, V. \& Wingreen, N. S. Responding to chemical gradients: bacterial chemotaxis. Curr. Opin. Cell Biol. 24, 262-268 (2012).

12. Lauga, E., DiLuzio, W. R., Whitesides, G. M. \& Stone, H. A. Swimming in circles: motion of bacteria near solid boundaries. Biophys. J. 90, 400-412 (2006).

13. Li, G. \& Tang, J. X. Accumulation of microswimmers near a surface mediated by collision and rotational Brownian motion. Phys. Rev. Lett. 103, 078101 (2009).

14. Zhang, H. P., Be'er, A., Florin, E.-L. \& Swinney, H. L. Collective motion and density fluctuations in bacterial colonies. Proc. Natl Acad. Sci. USA 107, 13626-13630 (2010).

15. Darnton, N. C., Turner, L., Rojevsky, S. \& Berg, H. C. Dynamics of bacteria swarming. Biophys. J. 98, 2082-2090 (2010).

16. Kohlmeier, S. et al. Taking the fungal highway: mobilization of pollutantdegrading bacteria by fungi. Environ. Sci. Technol. 39, 4640-4646 (2005).

17. Frey-Klett, P. et al. Bacterial-fungal interactions: hyphens between agricultural, clinical, environmental, and food microbiologists. Microbiol. Mol. Biol. Rev. 75, 583-609 (2011)

18. Pion, M. et al. Gains of bacterial flagellar motility in a fungal world. Appl. Environ. Microbiol. 79, 6862-6867 (2013).

19. Furuno, S. et al. Fungal mycelia allow chemotactic dispersal of polycyclic aromatic hydrocarbon-degrading bacteria in water-unsaturated systems. Environ. Microbiol. 12, 1391-1398 (2010).

20. Ingham, C. J., Kalisman, O., Finkelshtein, A. \& Ben-Jacob, E. Mutually facilitated dispersal between the nonmotile fungus Aspergillus fumigatus and the swarming bacterium Paenibacillus vortex. Proc. Natl Acad. Sci. USA 108, 19731-19736 (2011)

21. Pion, M. et al. Bacterial farming by the fungus Morchella crassipes. Proc. Biol. Sci. 280, 20132242 (2013).

22. Bravo, D. et al. Isolation of oxalotrophic bacteria able to disperse on fungal mycelium. FEMS Microbiol. Lett. 348, 157-166 (2013).

23. Worrich, A. et al. Mycelium-like networks increase bacterial dispersal, growth, and biodegradation in a model ecosystem at various water potentials. Appl. Environ. Microbiol. 82, 2902-2908 (2016).

24. Button, J. E. \& Dutton, R. J. Cheese microbes. Curr. Biol. 22, R587-R589 (2012).

25. Wolfe, B. E. \& Dutton, R. J. Fermented foods as experimentally tractable microbial ecosystems. Cell 161, 49-55 (2015).

26. Ropars, J., Cruaud, C., Lacoste, S. \& Dupont, J. A taxonomic and ecological overview of cheese fungi. Int. J. Food Microbiol. 155, 199-210 (2012).

27. Wolfe, B. E., Button, J. E., Santarelli, M. \& Dutton, R. J. Cheese rind communities provide tractable systems for in situ and in vitro studies of microbial diversity. Cell 158, 422-433 (2014).

28. Kastman, E. K. et al. Biotic interactions shape the ecological distributions of Staphylococcus species. MBio 7, e01157-16 (2016).

29. Irlinger, F. \& Mounier, J. Microbial interactions in cheese: implications for cheese quality and safety. Curr. Opin. Biotechnol. 20, 142-148 (2009).

30. Irlinger, F., Layec, S., Hélinck, S. \& Dugat-Bony, E. Cheese rind microbial communities: diversity, composition and origin. FEMS Microbiol. Lett. 362, 1-11 (2015).

31. Morin-Sardin, S., Rigalma, K., Coroller, L., Jany, J.-L. \& Coton, E. Effect of temperature, $\mathrm{pH}$, and water activity on Mucor spp. growth on synthetic medium, cheese analog and cheese. Food Microbiol. 56, 69-79 (2016).

32. Marcellino, N. \& Benson, D. R. The good, the bad, and the ugly: tales of moldripened cheese. Microbiol. Spectr. 1, 95-131 (2013).
33. Marcellino, S. N. \& Benson, D. R. Scanning electron and light microscopic study of microbial succession on Bethlehem St. Nectaire cheese. Appl. Environ. Microbiol. 58, 3448-3454 (1992).

34. Lund, F., Nielsen, A. B. \& Skouboe, P. Distribution of Penicillium commune isolates in cheese dairies mapped using secondary metabolite profiles, morphotypes, RAPD and AFLP fingerprinting. Food Microbiol. 20, 725-734 (2003).

35. Steinberg, G. Hyphal growth: a tale of motors, lipids, and the Spitzenkörper. Eukaryot. Cell 6, 351-360 (2007).

36. Hover, T. et al. Mechanisms of bacterial (Serratia marcescens) attachment to, migration along, and killing of fungal hyphae. Appl. Environ. Microbiol. 82, 2585-2594 (2016).

37. Kalbe, C., Marten, P. \& Berg, G. Strains of the genus Serratia as beneficial rhizobacteria of oilseed rape with antifungal properties. Microbiol. Res. 151, 433-439 (1996).

38. Kamensky, M., Ovadis, M., Chet, I. \& Chernin, L. Soil-borne strain IC14 of Serratia plymuthica with multiple mechanisms of antifungal activity provides biocontrol of Botrytis cinerea and Sclerotinia sclerotiorum diseases. Soil Biol. Biochem. 35, 323-331 (2003).

39. Jones, J. D., Grady, K. L., Suslow, T. V. \& Bedbrook, J. R. Isolation and characterization of genes encoding two chitinase enzymes from Serratia marcescens. EMBO J. 5, 467-473 (1986).

40. Wang, M., Xing, Y., Wang, J., Xu, Y. \& Wang, G. The role of the chil gene from the endophytic bacteria Serratia proteamaculans $336 \mathrm{x}$ in the biological control of wheat take-all. Can. J. Microbiol. 60, 533-540 (2014).

41. Neupane, S., Finlay, R. D., Alström, S., Elfstrand, M. \& Högberg, N. Transcriptional responses of the bacterial antagonist Serratia plymuthica to the fungal phytopathogen Rhizoctonia solani. Environ. Microbiol. Rep. 7, 123-127 (2015).

42. Goryshin, I. Y. \& Reznikoff, W. S. Tn5 in vitro transposition. J. Biol. Chem. 273, 7367-7374 (1998).

43. Galeva, A. et al. Bacterial flagellin-specific chaperone FliS interacts with antisigma factor FlgM. J. Bacteriol. 196, 1215-1221 (2014).

44. Mukherjee, S., Babitzke, P. \& Kearns, D. B. FliW and Flis function independently to control cytoplasmic flagellin levels in Bacillus subtilis. J. Bacteriol. 195, 297-306 (2013).

45. Yokoseki, T., Kutsukake, K., Ohnishi, K. \& Iino, T. Functional analysis of the flagellar genes in the fliD operon of Salmonella typhimurium. Microbiology 141, 1715-1722 (1995).

46. Yang, P., Zhang, M. \& van Elsas, J. D. Role of flagella and type four pili in the co-migration of Burkholderia terrae BS001 with fungal hyphae through soil. Sci. Rep. 7, 2997 (2017)

47. Gristwood, T., Fineran, P. C., Everson, L., Williamson, N. R. \& Salmond, G. P. The PhoBR two-component system regulates antibiotic biosynthesis in Serratia in response to phosphate. BMC Microbiol. 9, 112 (2009).

48. Shimizu, H., Nishiyama, K. \& Tokuda, H. Expression of $g p s A$ encoding biosynthetic sn-glycerol 3-phosphate dehydrogenase suppresses both the LBphenotype of a $\sec B$ null mutant and the cold-sensitive phenotype of a $\sec G$ null mutant. Mol. Microbiol. 26, 1013-1021 (1997).

49. Macnab, R. M. How bacteria assemble flagella. Annu. Rev. Microbiol. 57, 77-100 (2003)

50. Ohnishi, K., Fan, F., Schoenhals, G. J., Kihara, M. \& Macnab, R. M. The FliO, FliP, FliQ, and FliR proteins of Salmonella typhimurium: putative components for flagellar assembly. J. Bacteriol. 179, 6092-6099 (1997).

51. Cayenne Engel, E. \& Weltzin, J. F. Can community composition be predicted from pairwise species interactions? Plant Ecol. 195, 77-85 (2007)

52. Bleicher, A., Neuhaus, K. \& Scherer, S. Vibrio casei sp. nov., isolated from the surfaces of two French red smear soft cheeses. Int. J. Syst. Evol. Microbiol. 60 1745-1749 (2010).

53. Bowman, J. P. in The Prokaryotes (eds Dworkin, M. P., Falkow, S., Rosenberg, E., Schleifer, K.-H. \& Stackebrandt, E.) 920-930 (Springer, New York, 2006)

54. Manos, J. \& Belas, R. in The Prokaryotes (eds Dworkin, M. P., Falkow, S., Rosenberg, E., Schleifer, K.-H. \& Stackebrandt, E.) 245-269 (Springer, New York, 2006).

55. Vreeland R. H. in Bergey's Manual of Systematics of Archaea and Bacteria. (ed Whitman, W. B.) 1-19 (John Wiley \& Sons, Chichester, United Kingdom, 2015).

56. Janda, J. M. \& Abbott, S. L. The genus Hafnia: from soup to nuts. Clin. Microbiol. Rev. 19, 12-18 (2006).

57. Palleroni, N. J. in Bergey's Manual of Systematic Bacteriology, Vol. 1. (eds Krieg, N. R., Holt, J. G.) 141-199 (Williams \& Wilkins, Baltimore, Maryland, 1984).

58. Place, R. B., Hiestand, D., Gallmann, H. R. \& Teuber, M. Staphylococcus equorum subsp. linens, subsp. nov., a starter culture component for surface ripened semi-hard cheeses. Syst. Appl. Microbiol. 26, 30-37 (2003).

59. Brennan, N. M. et al. Microbacterium gubbeenense sp. nov., from the surface of a smear-ripened cheese. Int. J. Syst. Evol. Microbiol. 51, 1969-1976 (2001).

60. Irlinger, F., Bimet, F., Delettre, J., Lefèvre, M. \& Grimont, P. A. D. Arthrobacter bergerei sp. nov. and Arthrobacter arilaitensis sp. nov., novel coryneform 
species isolated from the surfaces of cheeses. Int. J. Syst. Evol. Microbiol. 55, 457-462 (2005).

61. Brennan, N. M. et al. Corynebacterium mooreparkense sp. nov. and Corynebacterium casei sp. nov., isolated from the surface of a smear-ripened cheese. Int. J. Syst. Evol. Microbiol. 51, 843-852 (2001).

62. Collins, M. D., Farrow, J. A., Goodfellow, M. \& Minnikin, D. E. Brevibacterium casei sp. nov. and Brevibacterium epidermidis sp. nov. Syst. Appl. Microbiol. 4, 388-395 (1983).

63. Whitman, W. et al. in Bergey's Manual of Systematic Bacteriology, Vol. 5 (Springer Science \& Business Media, Dordrecht, Netherlands, 2012).

64. Johnston, S. R., Boddy, L. \& Weightman, A. J. Bacteria in decomposing wood and their interactions with wood-decay fungi. FEMS Microbiol. Ecol. 92, fiw179 (2016).

65. Hoarau, G. et al. Bacteriome and mycobiome interactions underscore microbial dysbiosis in familial Crohn's disease. MBio 7, e01250-16 (2016).

66. Kalan, L. et al. Redefining the chronic-wound microbiome: fungal communities are prevalent, dynamic, and associated with delayed healing. MBio 7, e0105816 (2016).

67. Nseabasi, N. O. \& Antai, S. P. Effects of long-term kerosene spillage on heterotrophic microorganisms in soil from Niger Delta, Southern Nigeria. J. Appl. Sci. Environ. Manag. 16, 195-199 (2013).

68. Haug, R. H. Microorganisms of the nose and paranasal sinuses. Oral Maxillofac. Surg. Clin. North Am. 24, 191-196 (2012). vii-viii.

69. Findley, K. et al. Topographic diversity of fungal and bacterial communities in human skin. Nature 498, 367-370 (2013).

70. Aziz, R. K. et al. The RAST Server: rapid annotations using subsystems technology. BMC Genom. 9, 75 (2008).

71. Edgar, R. C. MUSCLE: multiple sequence alignment with high accuracy and high throughput. Nucleic Acids Res. 32, 1792-1797 (2004).

72. Stamatakis, A. et al. RAxML-Light: a tool for computing terabyte phylogenies. Bioinformatics 28, 2064-2066 (2012).

73. Hermet, A., Méheust, D., Mounier, J., Barbier, G. \& Jany, J.-L. Molecular systematics in the genus Mucor with special regards to species encountered in cheese. Fungal Biol. 116, 692-705 (2012).

74. Brennan, N. M. et al. Biodiversity of the bacterial flora on the surface of a smear cheese. Appl. Environ. Microbiol. 68, 820-830 (2002).

75. Valdés-Stauber, N., Scherer, S. \& Seiler, H. Identification of yeasts and coryneform bacteria from the surface microflora of brick cheeses. Int. J. Food Microbiol. 34, 115-129 (1997).

76. Frisvad, J. C. \& Samson, R. A. Penicillium subgenus Penicillium-A guide to identification of food and air-borne terverticillate Penicillia and their mycotoxins. Stud. Mycol. 49, 1-174 (2004).

77. David, L. A. et al. Diet rapidly and reproducibly alters the human gut microbiome. Nature 505, 559-563 (2014).

78. McClure, R. et al. Computational analysis of bacterial RNA-Seq data. Nucleic Acids Res. 41, el40 (2013).

79. Zhao, Y. et al. PGAP: pan-genomes analysis pipeline. Bioinformatics $\mathbf{2 8}$, 416-418 (2012).

\section{Acknowledgements}

This work was supported by the Tufts Collaborates Seed Grant Program from the Tufts University Office of the Provost and Senior Vice President (to B.E.W. and J.S.G.), the Knez Family Fund (to B.E.W.), the Tufts Russell L. Carpenter Fund (to Y.Z.), USDA NIFA grant 2017-67013-26520 (to B.E.W.), NSF grant 1715552 (to B.E.W.), NSF grant CAREER-1554095 (to J.S.G.), and NSF grant CBET-1511340 (to J.S.G.). We thank Esther Miller, Elizabeth Landis, Brittany Niccum, Fredrick Lee, Jason Shaffer, and Lucas Brown for providing feedback on earlier versions of this manuscript.

\section{Author contributions}

B.E.W. initiated, conceived, and supervised the project. J.S.G. initiated and supervised the cellular imaging. Y.Z. conducted all the experiments. E.K. performed the comparative genomics and other bioinformatic analyses in the paper. B.E.W., J.S.G., E.K., and Y.Z. wrote the paper.

\section{Additional information}

Supplementary Information accompanies this paper at https://doi.org/10.1038/s41467017-02522-Z.

Competing interests: The authors declare no competing financial interests.

Reprints and permission information is available online at http://npg.nature.com/ reprintsandpermissions/

Publisher's note: Springer Nature remains neutral with regard to jurisdictional claims in published maps and institutional affiliations.

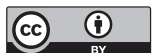

Open Access This article is licensed under a Creative Commons Attribution 4.0 International License, which permits use, sharing, adaptation, distribution and reproduction in any medium or format, as long as you give appropriate credit to the original author(s) and the source, provide a link to the Creative Commons license, and indicate if changes were made. The images or other third party material in this article are included in the article's Creative Commons license, unless indicated otherwise in a credit line to the material. If material is not included in the article's Creative Commons license and your intended use is not permitted by statutory regulation or exceeds the permitted use, you will need to obtain permission directly from the copyright holder. To view a copy of this license, visit http://creativecommons.org/ licenses/by/4.0/.

(C) The Author(s) 2018 